prognostic factors (18\%). Most use US to confirm clinical enthesitis when a spondyloarthropathy $(\mathrm{SpA})$ is suspected and half consider that a positive entheseal Dopppler signal supports more aggressive management. $76 \%$ perform real-time guided injections for the following disorders/sites: Baker's cyst, subacromial-subdeltoid bursa, tibionavicular joint, anterior coxofemoral joint, retrocalanceal bursa and extensor wrist tenosinovitis.

Abstract AB1190 - Table 1

\begin{tabular}{lc}
\hline & \% (Likert $\mathbf{4}$ \\
& $\mathbf{+ 5}$ \\
\hline To detect articular or periarticular inflammation when there is a clinical doubt & 94 \\
To detect enthesopathy, tendinosis or tendon ruptures when there is a & 79 \\
clinical doubt & \\
To detect crystal deposits when microcrystalline arthropathy is suspected & 48 \\
To detect bone erosions when radiology is obscure & 39 \\
To assess skin fibrosis & 35 \\
To assess palpable nodules or mases & 34 \\
To detect temporal artery vasculitis when it is clinically suspected & 20 \\
To detect salivary gland involvement in patients with xerostomy & 17 \\
To detect arteriosclerosis in chronic inflammatory patients & 13 \\
To assess interstitial lung disease & 2 \\
\hline
\end{tabular}

Conclusions: Rheumatologist- ultrasonographers in Spain use ultrasonography in a wide spectrum of diseases and clinical contexts mainly to facilitate diagnosis and improve treatment of rheumatic patients.

Disclosure of Interest: None declared

DOI: 10.1136/annrheumdis-2018-eular.4806

\section{AB1191 VITAMIN D AND CD34+ CELLS AS BIOMARKERS OF SUBCLINICAL ATHEROSCLEROSIS AND MYOCARDIAL DYSFUNCTION IN INFLAMMATORY JOINT DISEASES}

J. Rodríguez-Carrio $^{1,2}$, A. Lo Gullo ${ }^{3}$, F. Savarino ${ }^{3}$, C.O. Aragona ${ }^{3}$, G. Dattilo ${ }^{3}$, C. Zito ${ }^{4}$, A. Suárez ${ }^{1,2}$, S. Loddo ${ }^{3}$, M. Atteritano ${ }^{3}$, A. Saitta $^{3}$, G. Mandraffino ${ }^{3}$. ${ }^{1}$ Instituto de Investigación Sanitaria del Principado de Asturias (ISPA); ${ }^{2}$ Area of Immunology, University of Oviedo, Oviedo, Spain; ${ }^{3}$ Department of Clinical and Experimental Medicine, University of Messina, Messina, Italy; ${ }^{4}$ Department of Clinical and Experimental Medicine, University of Messina, Messina, Spain

Background: increased cardiovascular (CV) risk in inflammatory joint diseases (IJD) such as rheumatoid (RA) or psoriatic arthritis (PsA) is linked to an impaired vascular homeostasis. Chronic inflammation and immune dysregulation prompt endothelial damage and impair reparative mechanisms. Among them, circulating CD34 +bone marrow-derived progenitors are known to participate in endothelial turnover and improve myocardial neovascularization and ventricular remodelling, likely delaying CV disease development. Among factors related to CD34 +cells mobilisation, a role for vitamin $D$ has emerged in other scenarios. Whether impaired CD34 +cells or vitamin D levels underlie endothelial and myocardial dysfunction in IJD patients remains unknown.

Objectives: to evaluate the associations between CD34 + cells and vitamin D levels with markers of subclinical atherosclerosis and myocardial functionality in IJD patients.

Methods: CD34 + cells counts were assessed by flow cytometry in peripheral

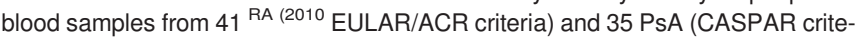
ria) patients recruited at onset and 58 matched healthy controls $(\mathrm{HC})$. Vitamin $D$ levels were quantified in serum by HPLC. PWV and cIMT were evaluated as markers of subclinical atherosclerosis, whereas myocardial dysfunction was assessed by speckle-tracking echocardiography (STE).

Results: vitamin D was decreased in RA (23.68 \pm 6.42$)$ and PsA $(23.53 \pm 4.84)$ compared to $\mathrm{HC}(31.75 \pm 5.05 \mathrm{ng} / \mathrm{ml}$, both $\mathrm{p}<0.001)$. Vitamin $\mathrm{D}$ was negatively associated with cIMT in $\mathrm{HC}(\mathrm{r}=-0.308, \mathrm{p}=0.026)$ and in PsA patients with low disease activity (DAS28 $<2.9, n=11)(r=-0.636, p=0.035)$, but not in their high disease activity or RA counterparts. CD34 +cells were decreased in RA compared to $\mathrm{HC}(1.58 \pm 0.58$ vs $2.35 \pm 1.14$ cells/ul, $\mathrm{p}=0.002)$. CD34 +cells frequency was associated with total- and LDL-cholesterol levels in $\mathrm{HC}(r=-0.295, p=0.031$ and $r=-0.354, p=0.011$, respectively) but not in IJD. CD34 +cells negatively paralleled DAS28 in PsA $(r=-0.338, p=0.047)$ and RA $(r=-0.303, p=0.057)$. Multivariate regression analyses revealed that vitamin $D$ levels $(B=0.019, p=0.009)$ and DAS28 $(B=-0.152, p=0.050)$ were independently associated with CD34 +counts in $\mathrm{PsA}$, whereas vitamin $\mathrm{D}(\mathrm{B}=0.010, p=0.028)$ and duration of the symptoms $(\mathrm{B}=0.016, \mathrm{p}=0.010)$ did in RA. CD34 +cells counts were correlated with GCS $(r=-0.291, p=0.068)$ and $G L S(r=-0.301, p=0.057)$ in $R A$.

Conclusions: vitamin D was negatively associated with cIMT and risk factors in $\mathrm{HC}$ and PsA patients with low disease activity, but not in those with active disease or RA. Vitamin D was an independent predictor of CD34 +cell depletion in IJD.
CD34 +cells, negatively associated with risk factors in $\mathrm{HC}$, were altered in RA in relation to disease activity and the duration of symptoms. CD34 +cells were associated with myocardial dysfunction in RA

Disclosure of Interest: None declared

DOI: 10.1136/annrheumdis-2018-eular.2435

\section{AB1192 DIAGNOSTIC YIELD OF MUSCLE BIOPSIES} PERFORMED OVER A 10 YEAR PERIOD

J.M. Weightman, K.A. Manchegowda, D. Coady. Rheumatology, Sunderland Royal Hospital, Sunderland, UK

Background: Patients with suspected inflammatory myopathy (IM) are often referred to Rheumatology, where the diagnostic process may include a biopsy of skeletal muscle. A new service was set up in 2007 at Sunderland Royal Hospital, whereby a trained consultant performs open muscle biopsies of vastus lateralis muscle under local anaesthetic within the Rheumatology outpatient department. Samples are sent to a histology laboratory at another hospital site for analysis, which can include electron microscopy. Referrals most commonly come from other consultant Rheumatologists within the team.

Objectives: To evaluate the diagnostic yield amongst muscle biopsies performed over a 10 year period. To review the correlation between final clinical diagnosis and investigation results. To identify any complications caused by the biopsy procedure.

Methods: Retrospective analysis of medical notes of all patients who were referred for muscle biopsy within the Rheumatology department during $2007-2017$.

Results: The mean patient age was 51 years; 28 patients were female. All procedures were performed or directly supervised by one trained rheumatology consultant. 45 patients were referred for muscle biopsy. 41 patients had elevated creatinine kinase. 2 were unable to tolerate the procedure. 3 samples were either too small for analysis or did not contain skeletal muscle. A total of 40 muscle samples were reviewed.

16 muscle biopsy samples showed histological features of IM (3 polymyositis, 3 dermatomyositis, 6 inclusion body myositis and 4 undifferentiated CTD). 15 samples showed other diagnoses including genetic, neurological and storage disorders. In 9 samples no definite diagnosis could be made on biopsy, despite this 3 patients were diagnosed with IM based on clinical features and other investigations.

Of the 19 patients with a final diagnosis of inflammatory myopathy (clinical and histological), 15 had positive ANA, 3 had negative ANA (1 of which had positive Ro antibodies).

EMG/NCS performed prior to muscle biopsy had a high positive predictive value: all 7 with an IM pattern on EMG had a histological diagnosis of IM. 2 patients with normal EMG had eventual diagnosis of IM

Complication rates were low. 3 patients had subsequent numbness around the biopsy site and 1 required a compression dressing for increased bleeding during the procedure.

Conclusions: Muscle biopsy was successful in achieving a diagnosis in $64 \%$ of all patients referred. Out of biopsies taken, $40 \%$ of biopsies performed showed IM. $37.5 \%$ showed other diagnoses. The total diagnostic yield is therefore calculated as $77.5 \%$. It appears to be a useful diagnostic investigation in patients with sus pected myopathies and helps with correct diagnosis and appropriate treatment. Muscle biopsy is relatively safe and can be performed in the outpatient setting Despite delays in the transfer of 3 specimens, histological analysis was still possible, suggesting that having an off-site histopathology laboratory does not adversely affect outcomes. Further review could focus on the increasing use of MRI scanning in the diagnostic evaluation of these patients, which may in some cases prevent the need for open biopsy.

Disclosure of Interest: None declared DOI: 10.1136/annrheumdis-2018-eular.5645

\section{$\mathrm{AB} 1193$ \\ AN IMMUNE REFERENCE ATLAS FROM BIRTH TO ADULTHOOD IDENTIFIES KEY DEVELOPMENTAL MILESTONES}

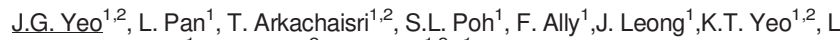
D/O Thana Bathi ${ }^{1}, A . Y . J$. Tan $^{2}$, S. Albani ${ }^{1,2}$. ${ }^{1}$ Translational Immunology Institute, SingHealth-DukeNUS Academic Medical Centre; ${ }^{2}$ KK Women's and Children's Hospital, Singapore, Singapore

Background: A developmental atlas of the immune system is key to understand ing its normal maturation process and identifying the disease-associated cell subsets. The absence of a holistic developmental immune normogram is a critical unmet need. 
Objectives: To address this need and our hypothesis that age-related immune effector and regulatory cell subsets changes are present, we employ a multidimensional approach using mass cytometry to identify the cell subsets that shape the developing immune landscape from birth to adulthood.

Methods: We interrogated the peripheral blood mononuclear cells from 126 healthy individuals (cord blood, newborn to adult) with mass cytometry using two extensive antibody panels embracing the most important cell lineages and their function. Quality control and batch effect correction were performed with outliers excluded before dimensional reduction and clustering to identify the unique immune cell subsets. Their frequencies across the age categories were presented as 3-D frequency histograms. Subsequent manual gating was done to further describe these immune cell subsets changes with age using Pearson correlation coefficient.

Results: Distinct developmental gradients involving multiple effector and regulatory cell subsets shaped the maturing immune landscape. The naïve TNF $\alpha+C D 4$ $+T$ cells were enriched in the early childhood period and declined with age (Pearson correlation coefficient, $r=-0.3503, p<0.001)$. In contrast, the memory TNF $\alpha$ $+C D 4+T$ cells increased with age $(r=0.4149, p<0.0001)$. A transitional milestone from naive to memory TNF $\alpha+C D 4+T$ cells was observed after 2 year old. This indicates that an intact CD4 +T cells TNF $\alpha$ effector mechanism is present at birth which progressively mature to involve the memory CD4 +T cells. For another cytokine IL17A, it was secreted solely by the memory CD4 $+\mathrm{T}$ cells with its population increasing with age $(r=0.3753, p<0.001)$. Another distinct maturation milestone was observed in the naive CD8 $+\mathrm{T}$ cell subset where a transition from IL8 to IFN $\gamma$ secretion after 10 year old was observed. The IL $8+$ and IFN $\gamma+$ naive CD8+T cell subsets correlated negatively and positively with increasing age respectively $(r=-0.2661, p<0.01$ and $r=0.2835, p<0.01)$.

For the $T$ regulatory cells, the age related changes were more gradual. The early thymic T regulatory cells (CD3+, CD4+, CD31+, CD45RA+, CD25+, Foxp3+, CD152+) demonstrated a gradual decline with age $(r=-0.2966, p<0.01)$ while the memory $T$ regulatory cells gradually increased with age (CD3+, CD4+, CD45RO ,+ CD25+, Foxp3+, CD152+) $(r=0.2444, p<0.05)$.

Conclusions: Key developmental milestones in the $T$ cell compartment were identified and with the other subsets identified, a holistic description of the developing immune landscape was obtained. This atlas has the potential dual translational role of defining the stage of immune maturity and distilling the pathological cell subset in both paediatric and adult immune mediated diseases. The database and the related pipeline to analyse it will be provided as a free reference.

Disclosure of Interest: None declared

DOI: 10.1136/annrheumdis-2018-eular.6099

\section{AB1194 ARE THERE NEW PARAMETERS TO BE CONSIDERED IN SPECTRAL DOPPLER TO EVALUATE THE NAIL BED IN PSORIASIS AND PSORIASIS ARTHRITIS?}

\section{J.A. Mendonça ${ }^{1}$, L.N. Pansani ${ }^{1}$, M.B. Mimoto ${ }^{1}$, E.C. Machado ${ }^{2}$, A.C. Bazan ${ }^{2}$. ${ }^{1}$ Rheumatology; ${ }^{2}$ Dermatology, PONTIFICAL CATHOLIC UNIVERSITY OF} CAMPINAS, Campinas, Brazil

Background: Other spectral Doppler parameters can assess joint impairment caused by psoriatic arthritis and psoriasis ${ }^{1}$.

Objectives: To detect and compare Doppler velocimetric indexes changes in 3 groups of patients.

Methods: Thirty - eight patients were evaluated: 8 in the control group - healthy (52 nail beds); 15 in the psoriasis group (134 nail beds); and 15 in the group with psoriatic arthritis (147 nail beds). "CASPAR" criteria were used to classify the patients. The ultrasound (US) was performed in all patients using Esaote MyLab 50 , with high resolution linear probe with a frequency of $18 \mathrm{mHz}$, it was positioned longitudinally to the nail bed.

Results: The psoriasis group of patients included 7 males (46.6\%) and 8 females (53.4\%); $66.6 \%$ were white, $33,4 \%$ black.

Abstract AB1194 - Table 1. Mean and standard deviations of US, clinical and laboratory variables by clinical group:

\begin{tabular}{lccc}
\hline & Psoriatic Arthritis & Psoriasis & Control \\
\hline $\mathbf{R I}($ Resistance Index) & $0.58(0.09)$ & $0.59(0.10)$ & $0.60(0.07)$ \\
$\mathbf{P I}($ Pulsatility Index) & $1.05(0.31)$ & $1.06(0.45)$ & $1.12(0.25)$ \\
Acceleration $\left(\mathbf{A}=\mathrm{m} / \mathrm{s}^{2} ;\right)$ & $0.37(0.29)$ & $0.39(0.47)$ & $0.51(0.44)$ \\
Acceleration Time $(\mathbf{A T}=\mathrm{ms})$ & $107.30(37.10)$ & $114.64(41.14)$ & $113.10(40.10)$ \\
Angle of Insonation & $18.21(25.8)$ & $14.76(24.71)$ & $27.28(30.10)$ \\
$\left(\boldsymbol{\alpha}={ }^{\circ}\right)$ & & & \\
Nail bed thickness & $1.60(0.78)$ & $1.68(0.50)$ & $1.44(0.40)$ \\
$(\mathbf{T}=\mathrm{mm})$ & & & \\
VHS & $27.5(24.9)$ & $20.4(14.9)$ & - \\
PCR & $0.75(1.1)$ & $0.54(0.7)$ & - \\
\hline
\end{tabular}

Abstract AB1194 - Table 2. Distribution and percentage of individuals according to clinical group and to the PASI, NAPSI variables:

\begin{tabular}{lccc}
\hline & Psoriatic Arthritis $\mathrm{N}(\%)$ & Psoriasis $\mathrm{N}(\%)$ & Control N $(\%)$ \\
\hline NAPSI & & & \\
Normal $(=0)$ & $0(0)$ & 0 & $8(100)$ \\
Mild $(<20)$ & $4(28,6)$ & $10\left(^{71,4}\right.$ & 0 \\
Severe $(>20)$ & $11\left({ }^{(68,7}\right.$ & $5(31,3)$ & 0 \\
PASI & & & 0 \\
Mild $(<15)$ & $14\left(^{51,8}\right.$ & $13\left(^{48,2}\right.$ & 0 \\
Moderate ${ }^{15-25}$ & $1(50,0)$ & $1(50,0)$ & 0 \\
Severe $(>25)$ & 0 & $1(100,0)$ & 0 \\
\hline
\end{tabular}

Statistically significant: Mean age and SD of the psoriasis, psoriatic arthritis and control groups, respectively: $57.13 \pm 14.3$ years; $54.66 \pm 10.6$ years; $24.87 \pm 2.03$ years $(p<0.05)$. A statistically significant difference was found in the NAPSI variable among all groups $(\mathbf{p}<0.05)$; in the variable PASI, a difference was only found in the control group $(\mathbf{p}<\mathbf{0 . 0 5})$ (Kruskall-Wallis) (table 2$)$; the A variable between the psoriasis group and control $(\mathbf{p}<\mathbf{0 . 0 0 1})$; between the psoriatic arthritis group and control $(\mathbf{p}=\mathbf{0 . 0 0 1})$, the variable $\mathbf{T}$ between the psoriasis and psoriatic arthritis groups $(p=0.006)$ and between the psoriasis and control groups $(p=0.001)$ and the $\alpha$ variable between control and psoriasis groups $(p=0.017)$ (table 1). Spearman and Pearson correlations between US variables per group, psoriasis, psoriatic arthritis and control were: RIxPI $r=0.744$ ( $p<0.001), P D$ (power Doppler) xT $r=0.301$ (p<0.001), RIxPI $r=0.914$ ( $p<0.001)$, PIxA $r=0.46(\mathbf{p}<0.001) ; R I x P I$ $r=0.889(p<0.001)$, PDxT $r=0.490(p<0.001)$ respectively.

Conclusions: There are other parameters in spectral Doppler to be validated in order to characterise changes in nail beds.

\section{REFERENCE:}

[1] Mendonça JA. High Specificity of Spectral Nail Assessment in Psoriatic Arthritis Patients [abstract]. Arthritis Rheumatol. 2015;67(suppl 10).

Disclosure of Interest: None declared

DOI: 10.1136/annrheumdis-2018-eular.3768

\section{AB1195 DEVELOPMENT AND VALIDATION OF AN ULTRASONOGRAPHIC ACTIVITY SCORE (USAS) FOR RHEUMATOID ARTHRITIS}

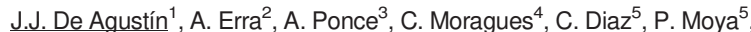
D. Reina ${ }^{6}$, P. Estrada 6 , E. Casado ${ }^{7}$, M. Moreno ${ }^{7}$, J. ramirez ${ }^{8}$, L. Mateo ${ }^{9}$, M. Pujol ${ }^{10}$, S. Ros ${ }^{11}$, P. Santo ${ }^{12}$, E.L. Sirvent ${ }^{12}$, J. Narvaez ${ }^{13}$, R. Sanmarti $1^{14}$, M. Garcia Yebenes ${ }^{15}$, L. Carmona ${ }^{15}$, on behalf of ECOCAT GROUP. ${ }^{1}$ Rheumatology Unit, Hospital Universitari Vall d'Hebron; ${ }^{2}$ Rheumatology, Hospital San Rafael, Barcelona; ${ }^{3}$ Rheumatology Unit, Hospital Granollers, Granollers; ${ }^{4}$ Rheumatology, Hospital Platon; ${ }^{5}$ Rheumatology, Hospital Sant Pau, Barcelona; ${ }^{6}$ Rheumatology, Hospital Moises Broggi, Sant Joan Despi; ${ }^{7}$ Rheumatology, Hospital Parc Tauli, Sabadell; ${ }^{8}$ Rheumatology, Hospital CLinic i Provincial, Barcelona; ${ }^{9}$ Rheumatology, Hospital Germans Trias i Pujol, Badalona; ${ }^{10}$ Rheumatology, Hospital de Tarrassa, Tarrassa; ${ }^{11}$ Rheumatology, Hospital de Viladecans, Viladecans; ${ }^{12}$ Rheumatology, Parc Sanitari Sant Joan de Deu, Sant Boi de Llobregat, ${ }^{13}$ Rheumatology, Hospital Universitari de Bellvitge, Hospitalet de Llobregat, ${ }^{14}$ Rheumatology, Hospital Clinic, BARCELONA; ${ }^{15}$ Inmusc, Instituto de Investigacion Musculoesqueletica, Madrid, Spain

Background: Composite scores developed in Rheumatoid arthritis (RA) not include all dimensions of disease activity. An index based on essential clinica plus a ultrasound (US) measures, focused on simplicity, with appropriate validation, would allow a better classification at different levels of disease activity than a clinical only or US only index.

Objectives: To develop and validate a mixed clinical-US inflammation score in RA for use in clinical practice.

Methods: Mixed methods. Experts elicited items reflecting inflammation which were prioritised by Delphi. Patients with RA with various grades of activity underwent clinical [28 swollen and tender joints counts, patient and physician globa assessment (PhGA), erythrocyte sedimentation rate, and $\mathrm{C}$-reactive protein (CRP)] and US assessments [synovitis or tenosynovitis by grey-scale (GS) and Power Doppler (PD) of 42 structures], blinded to the clinical assessment. An index was created after supported selection of US structures and scoring method. Construct validity was tested by correlation with DAS28, SDAI, CDAI, and PhGA. Reliability was evaluated in a subgroup of patients with the intraclass correlation coefficient (ICC).

Results: US of joints and tendons, CRP, and swollen joints were the items that passed the prioritisation phase. Then, 281 patients were randomly divided into design $(n=141)$ and validation analysis $(n=140)$. The combination of US sites chosen detected the maximum proportion of GS and PD present. Were elected 